\title{
Preface
}

Hamid Aghajan $^{\mathrm{a}}$, Aki Härmä ${ }^{\mathrm{b}}$, Kevin I-Kai Wang ${ }^{\mathrm{c}}$ and Juan Carlos Augusto ${ }^{\mathrm{d}}$

a Imec, IPI, Department of Telecommunications and Information Processing, Ghent University, Ghent, Belgium

${ }^{\mathrm{b}}$ Philips Research, Eindhoven, The Netherlands

c The University of Auckland, Auckland, New Zealand

${ }^{\mathrm{d}}$ Department of Computer Science and Group on Development of Intelligent Environments, Middlesex University, London, UK

\section{This issue}

This issue of JAISE is composed of four papers. The review process for the papers in this issue was supervised by our editors Egon L. van den Broek, Jinshi Cui, Fabio Paterno, and Caifeng Shan, whom we thank for their service.

Mobile robots providing services in smart homes need to be guided to learn the boundaries of different workspaces. Virtual borders defined by the user are often used to restrict a robot's mobility in humancentered environments, e.g. to define privacy zones and areas for work. However, current methods of defining zones heavily depend on the robot's on-board sensing capabilities and often require a direct line of sight between the human user and the robot. The paper "Interactive restriction of a mobile robot's workspace in a smart home environment" by Sprute et al. proposes an interactive method based on employing multiple sensory types including cameras and laser pointers to extract virtual borders through interactions between the human user and the robot. Shorter interaction time and better user experience are supported by the proposed technique.

One of the challenges in human activity recognition is to distinguish activities that have infrequent occurrences and possess less distinctive patterns in presence of regular activities that occur frequently. The paper "Representation learning for minority and subtle activities in a smart home environment" by Sanabria et al. proposes a hierarchical activity classifier based on a two-phase learning scheme. In the first phase the classifier learns general features to recognise major types of activities, and in the second phase it collects minority and subtle activity types and identifies fine differences between them. A feature of the proposed approach is to reduce the bias of the ensemble classifier by training it on a subspace of data, hence allowing the identification of activities with subtle differences.

With the advent of smart computing, Internet of Things (IoT), and sensor technology, many applications in monitoring the wellness of elderly users living alone in a home have been studied in recent years. By employing miniaturized sensors, it is possible to monitor and label the situation of the user as normal or abnormal depending on the sensor reports and contextual factors. Despite the significant progress made in this area, there is still a need for the development of new classification techniques to capture various aspects of activities necessary for predicting wellness with high accuracy. The paper "Wellness determination of the elderly using spatio-temporal correlation analysis of daily activities" by Ujager et al. proposes a classification model by integrating the spatiotemporal contextual information for predicting wellness of an elderly user by monitoring their daily usage patterns of household appliances equipped with sensors. The method generates classification rules by identifying correlations between frequent and less frequent activities recorded over predefined time windows. The proposed model learns from new data and updates activity rules dynamically to accommodate changes in the daily activity patterns, and hence offers adaptivity with daily routine changes over time.

The emerging area of predictive health analytics offers great promises in reducing costs and improving health outcomes of patients suffering from chronic illnesses. Most predictive models do not capture environmental exposures that may impose health risk patterns in a particular illnesse. The paper "Predictive and exposome analytics: A case study of asthma exacerbation management" by Alkobaisi et al. pro- 
poses a framework for improving preventive interventions through meaningful and understandable feedback on the collected data from the individual and minimizing their exposure to health risks. The proposed method focuses on the simultaneous monitoring of the mobility behaviour of the patient and the measurement of environmental conditions to capture their impact on the user's health. Managing the risks associated with asthma symptoms are used as a case study to evaluate the effectiveness of the proposed spatiotemporal analysis of the data in the model training process.

\section{Upcoming issues}

The following is the list of upcoming issues of JAISE:
- January 2020: Thematic Issue on "Artificial Intelligence for IoT Systems"

- March 2020: Regular Issue

- May 2020: Thematic Issue on "Selected Papers from Intelligent Environments 2019"

- July 2020: Regular Issue

- September 2020: Thematic Issue on "Human Autonomous Devices for Rehabilitation and Assistance"

- November 2010: Regular Issue

More information on the call for papers for future thematic issues is available on the webpage of JAISE at: http://www.iospress.nl/journal/journal-of-ambientintelligence-and-smart-environments/ 